\title{
Continuous poliglecaprone suture versus bidirectional barded suture for urethrovesical anastomosis during laparoscopic radical prostatectomy: a single-institute retrospective analysis of Chinese patients
}

\author{
Jiaxing Li ${ }^{1}$, Qi Zhang ${ }^{2}$, Jianwei Xie ${ }^{1}$, Bo Yin ${ }^{1}$ \\ ${ }^{1}$ Department of Urology, ${ }^{2}$ Department of Ultrasound, Shengjing Hospital of China Medical University, Shenyang, China \\ Contributions: (I) Conception and design: B Yin; (II) Administrative support: B Yin; (III) Provision of study materials or patients: B Yin, J Li; (IV) \\ Collection and assembly of data: J Xie, J Li; (V) Data analysis and interpretation: J Li, Q Zhang; (VI) Manuscript writing: All authors; (VII) Final \\ approval of manuscript: All authors. \\ Correspondence to: Bo Yin. Department of Urology, Shengjing Hospital of China Medical University, No. 36 SANHAO Road, Shenyang 110004, \\ China. Email: yinb@sj-hospital.org.
}

\begin{abstract}
Background: To compare the outcomes of bidirectional barbed suture and continuous poliglecaprone suture for urethrovesical anastomosis (UVA) during laparoscopic radical prostatectomy (LRP).

Methods: Single-needle poliglecaprone suture was used for UVA in Group 1 ( $n=27)$, and double-needle bidirectional barbed suture was used for UVA $(n=27)$ in Group 2. Age, body mass index (BMI), prostatespecific antigen (PSA) level, prostate volume, operative time, anastomosis time, estimated blood loss, Gleason score, number of hospitalization days, and urinary continence at postoperative month 1, 3, 6, and 12 were analyzed statistically.
\end{abstract}

Results: There were no significant differences in age, BMI, prostate volume, PSA level, Gleason score, and indwelling catheter time between the two groups. However, bidirectional barbed suture was associated with a significantly shorter anastomosis time $(\mathrm{P}=0.007)$, operation time $(\mathrm{P}=0.008)$ and hospitalization duration $(\mathrm{P}<0.001)$, and a significantly lower blood loss volume $(\mathrm{P}=0.005)$. At the first-month followup, urinary continence was achieved in $5(19 \%)$ and $15(56 \%)$ patients in Group 1 and 2, respectively $(\mathrm{P}=0.005)$; postoperative third month: $11(41 \%)$ and $23(85 \%)$ patients in Group 1 and 2 respectively $(\mathrm{P}=0.001)$; postoperative sixth month: $21(78 \%)$ and $25(93 \%)$ patients in Group 1 and 2 respectively $(\mathrm{P}=0.250)$; postoperative first year: 25 (93\%) and $27(100 \%)$ patients in Group 1 and 2 respectively $(\mathrm{P}=0.471)$. Multivariate regression analysis showed that anastomosis time $(\mathrm{HR}=0.636 ; \mathrm{P}<0.001)$ was an independent predictor of postoperative continence.

Conclusions: The current findings show that bidirectional barbed suture for UVA during LRP can shorten UVA time and provide better outcomes in terms of early urinary continence recovery.

Keywords: Laparoscope; radical prostatectomy; urinary continence; barbed suture; urethrovesical anastomosis

Submitted Mar 19, 2020. Accepted for publication Jul 31, 2020.

doi: 10.21037 /gs-20-371

View this article at: http://dx.doi.org/10.21037/gs-20-371

\section{Introduction}

Prostate cancer is the most common solid organ malignancy in the male population, and it is also the male malignant tumor with the second highest incidence rate and sixth highest mortality rate globally (1). In China, in 2014, prostate cancer was the male urinary tumor with the highest incidence $(98 / 100,000)$ and mortality $(4.22 / 100,000)(2)$. Further, 60,300 new cases of prostate cancer and 26,600 
new prostate cancer-related deaths were reported in China in 2015 (3). The incidence of and mortality associated with this cancer has been on the increase in China.

Radical prostatectomy (RP) remains the standard surgical treatment for long-term control of clinically localized prostate cancer. As an advanced medical technology, robot-assisted RP (RARP) has tremendously changed the art of performing prostatectomy, but laparoscopic RP (LRP) is still routinely performed for localized prostate cancer at many centers that do not have a robot. Urinary incontinence is one of the most dreaded complications that influences the quality of life of patients who have undergone $\mathrm{RP}$. One of the factors associated with this complication is urethrovesical anastomosis (UVA), which is the final step of the procedure. However, laparoscopic intracorporeal suturing for achieving UVA is one of the most challenging and time-consuming tasks for surgeons. Therefore, the surgical techniques for this part of the procedure have seen continuous improvements over the years (4).

The standard method for UVA, which involves a poliglecaprone suture, requires follow-through by an assistant when continuous anastomosis is performed. An assistant in training may find it difficult to follow through; this may affect the quality of the anastomosis, and thereby result in complications. As a solution to the issues with the poliglecaprone sutures, barbed sutures were introduced: these self-anchoring knotless sutures incorporate tiny barbs spaced evenly in a helical array on the suture. The advantages of barbed sutures are that they prevent slipping and ensure even distribution of tissue force across different barb points. In China, the continuous poliglecaprone suture method has been widely adopted; in particular, the single-needle continuous anastomosis method advocated by Zhang (5) has been shown to have several advantages. We have tried to improve this method with the use of a double semi-circular anastomosis technique called double-needle bidirectional barbed suture. The present study is a singleinstitute retrospective analysis of the use of poliglecaprone suture and bidirectional barded suture for UVA during LRP. We present the following article in accordance with the STROBE reporting checklist (available at http://dx.doi. org/10.21037/gs-20-371).

\section{Methods}

\section{Study participants}

Between 2013 and 2019, a total of 54 consecutive patients underwent LRP that was performed by one experienced surgeon at a single medical center. The study cohort included 27 consecutive patients who underwent UVA with the poliglecaprone suture and 27 consecutive patients who underwent UVA with bidirectional barded suture. The inclusion criteria were clinically organ confined or locally advanced prostate cancer. Patients who had undergone prostatic, urethral, or bladder neck surgery or neoadjuvant therapy previously, as well as those with indications of extracapsular extension on magnetic resonance imaging and those with preoperative incontinence were excluded. All the patients underwent pelvic magnetic resonance imaging before the procedure. The study was conducted in accordance with the Declaration of Helsinki (as revised in 2013). The study was approved by the ethics committee of Shengjing Hospital of China (NO. 2020PS059K) Medical University and individual consent for this retrospective analysis was waived.

\section{Characteristics of the study population}

Baseline patient characteristics were recorded at the preoperative clinic visit, including age at surgery, BMI, last preoperative prostate-specific antigen (PSA) level, and prostate volume.

Surgical and postoperative parameters were recorded, including operative time, anastomosis time, estimated blood loss, postoperative Gleason score (Gleason Score was grouped by the Prostate Cancer Grade Group system), and hospitalization days.

Patients were managed routinely after the procedure, and Foley catheter removal was performed at postoperative days 14-21. Follow-up data were available for patients who returned for a postoperative clinic visit and telephone visit, which typically occurred at postoperative month 1, 3, 6, and 12. At each follow-up visit, patients were asked about the number of pads used per day, and PSA was measured at 3 -month intervals.

\section{Study endpoints}

Postoperative urinary incontinence and sexual function are the main concerns of patients with prostate cancer. However, for Chinese patients, sexual function is rarely concerned, then the recovery of urinary continence means the main functional outcome. Thus, we chose the recovery of urinary continence as the endpoint of our study. Patients without urine leakage in their daily life, those who used only 


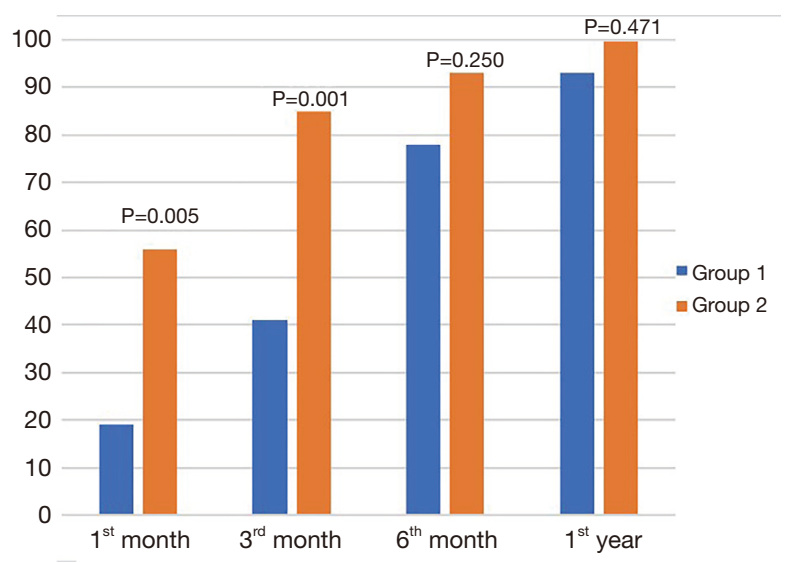

Figure 1 Clustered column chart showing the comparison between Group 1 and Group 2 continence rates at the $1^{\text {st }}$ month, $3^{\text {rd }}$ month, 6 th month and $1^{\text {st }}$ year after the procedure.

one pad per day for safety reasons, and those who did not have any limitations in their daily activities were considered to have achieved urinary continence.

\section{Surgical technique}

LRP was performed via the conventional transperitoneal approach combined with the anterior approach.

The transperitoneal approach involved separating the seminal vesicles from the posterior wall of the prostate, dissociating the seminal vesicles and the vas deferens, and dissecting the Denonvilliers fascia via the posterior approach. The anterior approach involved separating the anterior wall of the bladder, extending the Retzius space, opening the endopelvic fascia on both sides, dissecting the puboprostatic ligament and suturing the dorsal vascular complex, making an incision in the anterior wall of the bladder neck, disconnecting the posterior wall of the bladder neck, separating the bilateral vascular bundles of the prostate, sparing the neurovascular bundle, dissociating the apex of the prostate, and dissecting the posterior urethra (while preserving the length of the posterior urethra as much as possible). Finally, UVA via bidirectional barded suture or poliglecaprone suture was performed.

For Group 1, a 30-cm 2-0 poliglecaprone suture with a 26-mm 1/2C needle was used. The first suture was placed at the 8 o'clock position-outside-in on the bladder neck and inside-out on the urethra-and then the knot was tied. Next, the anastomosis was continued in a clockwise fashion, and the suture was run to the initial 8 o'clock position.
For Group 2, a $14 \times 14 \mathrm{~cm} \mathrm{2-0} \mathrm{bidirectional} \mathrm{knotless}$ barded suture (ETH105 V002) with two 26-mm 1/2C needles was used. Five trocars were used (Figure 1): a left lateral trocar in the left hand, a right medial trocar in the right hand. For the last stitch on the right, the left medial trocar in the right hand was used. The first suture was placed on the posterior lip of the bladder neck outside-in and inside-out on the posterior wall of the urethra, under the guidance of the ureter. Then, a mucosa-to-mucosa suture was performed at low tension at 5.3 o'clock with a forward needle, and at 7.9 o'clock with a reverse needle. Following this, the suture was tightened, the bladder neck and urethra were aligned, and the urinary catheter was replaced with a fresh one. The suture was continued in a similar way at 1 and 11 o'clock, until the two arms met at 12 o'clock, without the need for a knot.

A $100-\mathrm{mL}$ normal saline bladder filling was used at the end of all the procedures in order to rule out any leakage.

\section{Statistical analysis}

Functional data of all the patients were surveyed during routine outpatient and telephone follow-up, and then retrospectively collected for statistical analysis. Statistical analysis was performed using the Statistical Software for Social Sciences, version 22.0 (SPSS 22.0; IL, USA).

Continuous variables were presented as mean or median, and categorical variables were presented as percentages.

Continuous variables with normal distribution were compared with the Independent sample $t$-test, and data that were not normally distribution were compared between groups with the Mann-Whitney $U$-test. In addition, the chi-square and Fisher exact tests were used to compare categorical variables. $\mathrm{P}$ values less than 0.05 were considered to indicate significance. A multivariate logistic regression model was used to predict the association between early recovery of urinary continence and the analyzed variables.

\section{Results}

The baseline clinical, pathological, and operative characteristics of the patients according to anastomotic suture type are summarized in Table 1. Of the 54 patients in the cohort, bidirectional barbed suture was used in 27 $(50 \%)$ patients, and poliglecaprone continuous suture was used in $27(50 \%)$ patients. There were no significant differences between these two groups with regard to patient age, BMI, prostate volume, last preoperative PSA 
Table 1 Comparison of surgical and postoperative parameters between the two groups, mean (median)

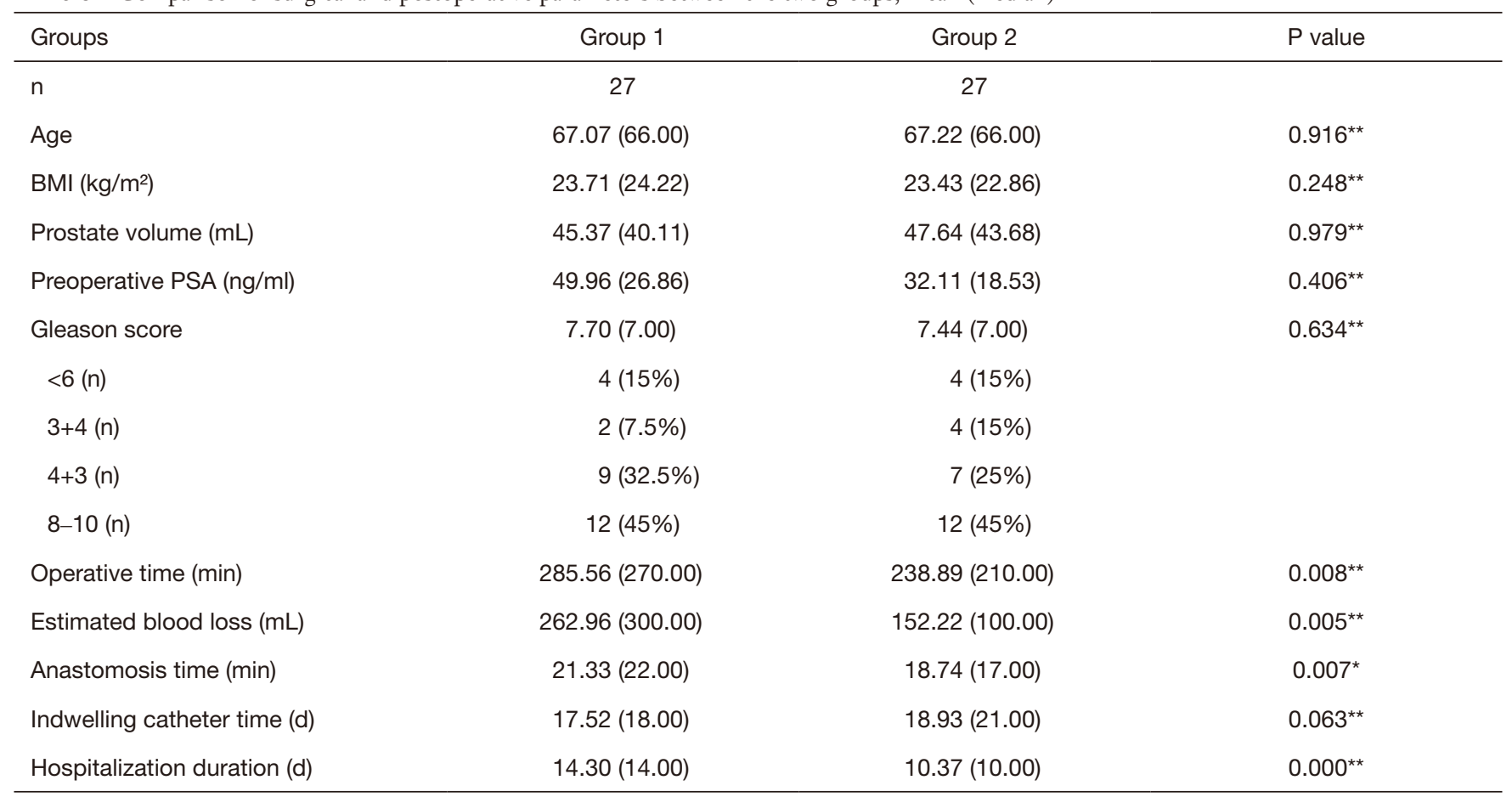

*group 1 vs. group 2, as per the independent sample $t$-test; ** group 1 vs. group 2, as per the Mann-Whitney $U$-test. BMI, body mass index; PSA, prostate-specific antigen. Group 1: Single-needle poliglecaprone suture; Group 2: Double-needle bidirectional barbed suture.

Table 2 Comparison of postoperative urinary continence recovery between the two groups

\begin{tabular}{|c|c|c|c|}
\hline Groups & Group 1 & Group 2 & $P$ value \\
\hline Postoperative urinary continence at 1 st month, $n$ [\%] & $5[19]$ & $15[56]$ & $0.005^{\star \star \star}$ \\
\hline Postoperative urinary continence at 3th month, $\mathrm{n}[\%]$ & $11[41]$ & 23 [85] & $0.001^{\star \star *}$ \\
\hline Postoperative urinary continence at 6th month, $\mathrm{n}[\%]$ & $21[78]$ & 25 [93] & $0.250^{\star \star \star}$ \\
\hline
\end{tabular}

${ }^{* * *}$ group 1 vs. group 2, by the Chi-square test. Group 1: Single-needle poliglecaprone suture; Group 2: Double-needle bidirectional barbed suture.

level, Gleason score, and indwelling catheter time $(\mathrm{P}>0.05)$. With regard to complications, there were two cases of postoperative urine leakage (1 case of groups), both of which were cured by conservative treatment. There were also two cases ( 1 case of groups) of urethral stricture that were treated with dilatation. However, bidirectional barbed suture required significantly less anastomosis time than poliglecaprone continuous suture (21.33 vs. $18.74 \mathrm{~min}$, $\mathrm{P}=0.007)$. Moreover, the operative time was significantly shorter in the bidirectional barbed suture group (270.00 vs. $210.00 \mathrm{~min}, \mathrm{P}=0.008$ ), which also had a significantly shorter hospitalization duration (10.00 vs. 14.00 days, $\mathrm{P}<0.001)$ and significantly lower blood loss volume $(100.00$ vs. $300.00 \mathrm{~mL}, \mathrm{P}=0.005)$ than the continuous suture group.

Postoperative urinary continence recovery rates were generally higher in the bidirectional barbed suture group than in the continuous suture group: it was significantly higher in the bidirectional barbed suture group at postoperative month $1(56 \%$ vs. $19 \%, \mathrm{P}=0.005)$ and postoperative month 3 ( $85 \%$ vs. $41 \%, \mathrm{P}=0.001$ ) (Table 2 \& Figure 2). However, the difference was not statistically 
significant at postoperative month 6 (93\% vs. $78 \%$, $\mathrm{P}=0.250)$ and postoperative month 12 (100\% vs. $93 \%$, $\mathrm{P}=0.471)$.

According to the findings of multivariable Cox regression analyses (Table 3), after controlling for all potential confounders, anastomosis time $(\mathrm{HR}=0.636, \mathrm{P}<0.001)$ was found to be an independent predictor of postoperative continence recovery after LRP.

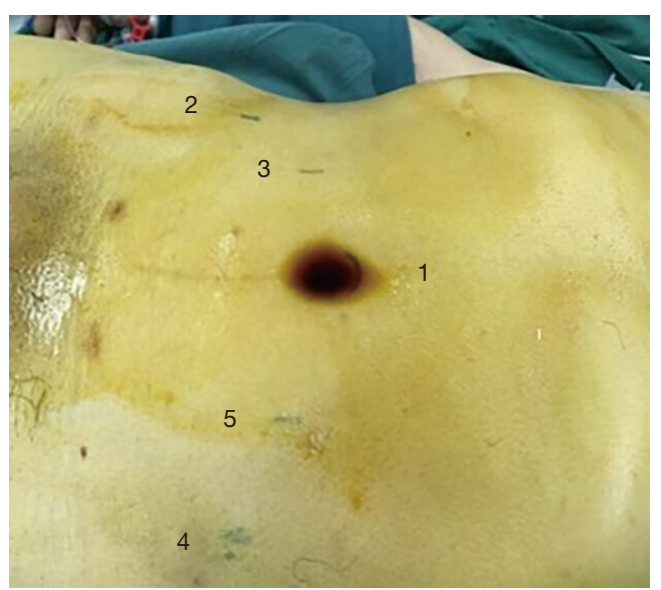

Figure 2 The locations of all the trocars. 1: Superior/inferior median umbilicus; 2 and 4: Medial side of the anterior superior iliac spine; 3 and 5: The outer margin of the rectus abdominis.

\section{Discussion}

The present study compares bidirectional barbed suture (a new technique for UVA) and continuous poliglecaprone suture (a standard technique for UVA) in a single-institute cohort of patients from China. This is the first study to compare these two methods in patients from China.

In this study, double-needle bidirectional barbed suture had several advantages over continuous suture, as the former was associated with a significantly shorter operation time, anastomosis time, and length of hospital stay (Table 1). Similar to the present findings, Lin et al.'s (6) meta-analysis of 12 studies on barbed sutures reported that the technique had a shorter anastomosis time, operation time, and hospitalization time. Similarly, Hemal et al. (7) found that the barbed suture group had significantly shorter anastomosis time and hospital stay, and Cakici et al. (8) reported that the anastomosis time of the barbed suture group was significantly shorter than that of the continuous suture group. Thus, the studies seem to generally agree that the barbed suture technique has a shorter anastomosis, hospitalization, and procedure duration.

In the current study, the barbed suture technique resulted in lower blood loss volume than the continuous suture method. Similarly, Porreca et al. (9) found that the barbed suture technique reduced bleeding and anastomotic leakage. The bidirectional barbed suture technique provides

Table 3 Univariate and multivariable Cox logistic regression analysis for predicting the independent variable for urinary continence recovery after LRP

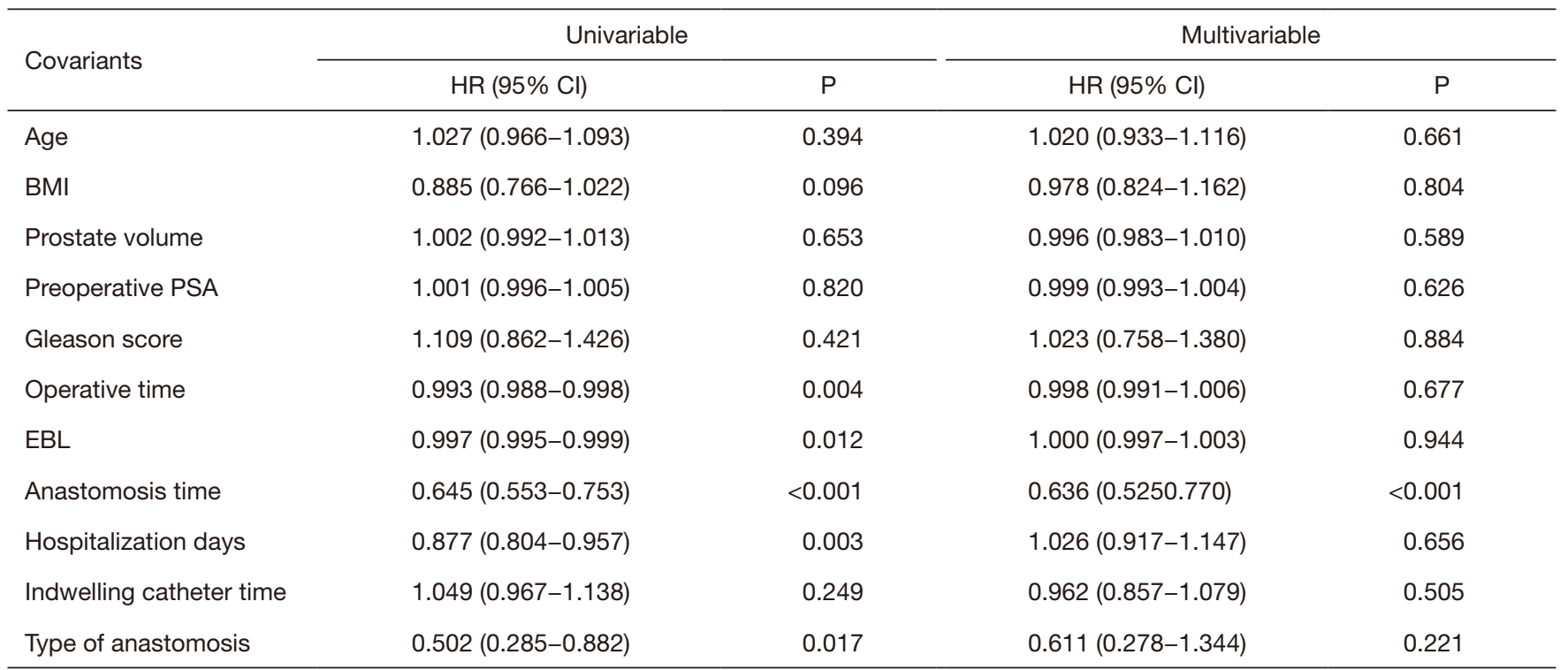

$\mathrm{Cl}$, confidence interval; HR, hazard ratio; BMI, body mass index; PSA, prostate-specific antigen; EBL, estimated blood loss. 
secure closure of the incision by distributing tension evenly across the anastomotic stoma and preventing any sliding of the suture, thereby reducing the chances of anastomotic leakage and helping the anastomosis heal better. Further, compared to the conventional continuous suture, the bidirectional design precludes the need for assistance or knot tying, improves anastomotic efficiency, and reduces the anastomotic time and operation time. However, it is unclear whether the intraoperative blood volume loss is directly associated with the UVA technique used.

In this study, the complication rates were similar in both groups: there was one case each of urethral stricture and urine leakage. Compared with the two studies of Ouzaid et al. (10) and Jacobsen et al. (11), the incidence of urethral stricture in the double-needle bidirectional barbed suture group was low. The reason for the difference is that bidirectional barbed suture prevents slipping. In the current cases, urinary stricture was treated with dilatation. As a preventive measure, the indwelling catheter can be left in for an extended postoperative period to reduce the chances of urethral stricture (12).

With regard to the postoperative urinary continence rates, in this study, double-needle bidirectional barbed suture resulted in significantly higher urinary continence recovery rates than the single-needle continuous suture at the postoperative first and third month. The recovery rate was higher in the sixth month and first year after surgery, too, but the difference was not significant. In agreement with our findings, the study by Ye and colleagues reported similar urinary continence rates for UVA with knotless bidirectional barbed suture at the postoperative third month, sixth month, and first year (13). Further, De Carlo and coworkers (14) and Zhang and co-workers (15) also reported urinary continence rates that were similar to those of the double-needle bidirectional barbed group of the present study at the third month, sixth month, and first year after RARP in their retrospective studies. The advantage of barbed suture over single-needle poliglecaprone continuous suture group in terms of continence recovery was also reported in a prospective study by Takeda and colleagues (16). Additionally, Cakici et al. (8) reported significantly higher urinary continence rates at the postoperative first month in the bidirectional barbed suture group. We considered that possible reasons may be: First of all, the single-needle continuous suture method is difficult to achieve even force in the whole circle when it is pulled from a physical point of view, so the anastomosis can't be well done. Secondly, the pulling of double-needle bidirectional barbed suture from the starting point to both sides of the anastomotic site makes the tension evenly distributed at the anastomotic site and prevents the anastomotic site from sliding and twisting, which is conducive to the healing of the anastomotic site. It should be noted that although all these studies reported that the barbed suture technique was more advantageous, the urinary continence rates reported were different at the same time points after surgery. This difference could be attributed to the subjective assessment of this condition based on the number of pads used daily, as it is not possible to control when patients change, the size of the pads, and whether each pad is filled with urine before it is replaced. Other contributing factors could be individual differences between patient conditions, ethnic differences, preservation of the vascular nerve bundles, the length of the preserved posterior urethra, ligation of the retropubic vascular complex, and other differences in techniques.

Barbed sutures also have some disadvantages. For example, when the suture is overtightened, the barbs could "grasp" the surrounding tissue, block blood flow, and cause secondary injury. Williams et al. (17) reported in a prospective randomized controlled study that hematuria symptoms occurred on the fifth day after surgery with the barbed suture technique; cystographic analysis showed that tissue necrosis might be the cause of injury and hematuria. Further, a prospective cohort study (18) showed that urethral injuries and paraurethral tissue injuries occurred in $58.3 \%$ and $45.8 \%$ of the patients in the barbed suture group, respectively, and these values were significantly higher than those $(21.6 \%$ and $18.9 \%$ respectively) in the non-barbed suture group. This might be a result of reduction of the elasticity of the external urethral sphincter and the subsequent continuous deterioration of external urethral sphincter function. Although such complications did not occur in the present cohort, this is an important subject of research for the future.

The main limitations of the present study are the small sample size and retrospective design. Besides, because of the different methods during two periods, the surgeon's technique would have been improved during the second period. In this case, it may affect the surgical results of the two methods. Additionally, we did not report about improvement of virility, because the majority of the elderly population in Asia are more concerned about continence than intercourse. Another limiting factor is that urinary continence can only be determined based on the patient's subjective judgment of the number of pads used daily, and 
not based on objective indicators. Therefore, a prospective study or another retrospective cohort study with a large sample is required to confirm the current findings.

\section{Conclusions}

The findings of this retrospective study imply that the double-needle bidirectional barbed suture technique provides excellent functional outcome without compromising on oncological control, and is safe and feasible for LRP. However, the findings need to be verified in a large sample as the current sample size was rather small.

\section{Acknowledgments}

Funding: 345 Talent Project of Shengjing Hospital of China Medical University.

\section{Footnote}

Reporting Checklist: The authors have completed the STROBE reporting checklist. Available at http://dx.doi. org/10.21037/gs-20-371

Data Sharing Statement: Available at http://dx.doi. org/10.21037/gs-20-371

Conflicts of Interest: All authors have completed the ICMJE uniform disclosure form (available at http://dx.doi. org/10.21037/gs-20-371). The authors have no conflicts of Interest to declare.

Ethical Statement: The authors are accountable for all aspects of the work in ensuring that questions related to the accuracy or integrity of any part of the work are appropriately investigated and resolved. The study was approved by the Ethics Committee of Shengjing Hospital of China Medical University (number of ethical approval: 2020PS059K). The study was conducted in accordance with the Declaration of Helsinki (as revised in 2013). Informed consent was not required since this was a retrospective study and all data with personal identifiers were kept confidential.

Open Access Statement: This is an Open Access article distributed in accordance with the Creative Commons Attribution-NonCommercial-NoDerivs 4.0 International License (CC BY-NC-ND 4.0), which permits the noncommercial replication and distribution of the article with the strict proviso that no changes or edits are made and the original work is properly cited (including links to both the formal publication through the relevant DOI and the license). See: https://creativecommons.org/licenses/by-nc-nd/4.0/.

\section{References}

1. Saracci R, Wild CP. Fifty years of the International Agency for Research on Cancer (1965 to 2015). Int J Cancer 2016;138:1309-11.

2. Chinese guidelines for diagnosis and treatment of prostate cancer 2018 (English version). Chin J Cancer Res 2019;31:67-83.

3. Chen $\mathrm{W}$, Zheng R, Baade PD, et al. Cancer statistics in China, 2015. CA Cancer J Clin 2016;66:115-32.

4. Juan Escudero JU, Ramírez Backhaus M, Benedicto Redón A, et al. Use of a barbed suture for the urethro-vesical anastomosis during the learning curve of the endoscopic extraperitoneal radical prostatectomy. Arch Esp Urol 2012;65:752-8.

5. Zhang X QZ, Wang C, Ai X. Single needle running suture method for urethrovesical anastomosis during laparoscopic radical prostatectomy. Chin J Urol 2009;30:476-9.

6. Lin YF, Lai SK, Liu QY, et al. Efficacy and safety of barbed suture in minimally invasive radical prostatectomy: A systematic review and meta-analysis. Kaohsiung J Med Sci 2017;33:107-15.

7. Hemal AK, Agarwal MM, Babbar P. Impact of newer unidirectional and bidirectional barbed suture on vesicourethral anastomosis during robot-assisted radical prostatectomy and its comparison with polyglecaprone- 25 suture: an initial experience. Int Urol Nephrol 2012;44:125-32.

8. Cakici OU, Canda AE. Bidirectional Barbed Only vs Poliglecaprone Suture with Rhabdosphincter Reconstruction for Urethrovesical Anastomosis During Robotic Radical Prostatectomy: Does It Make Any Difference? J Endourol 2018;32:944-9.

9. Porreca A, D'Agostino D, Dandrea M, et al. Bidirectional barbed suture for posterior musculofascial reconstruction and knotless vesicourethral anastomosis during robotassisted radical prostatectomy. Minerva Urol Nefrol 2018;70:319-25.

10. Ouzaid I, Xylinas E, Ploussard G, et al. Anastomotic stricture after minimally invasive radical prostatectomy: what should be expected from the Van Velthoven singleknot running suture? J Endourol 2012;26:1020-5.

11. Jacobsen A, Berg KD, Iversen P, et al. Anastomotic 
complications after robot-assisted laparoscopic and open radical prostatectomy. Scand J Urol 2016;50:274-9.

12. Albisinni S, Aoun F, Peltier A, et al. The Single-Knot Running Vesicourethral Anastomosis after Minimally Invasive Prostatectomy: Review of the Technique and Its Modifications, Tips, and Pitfalls. Prostate Cancer 2016;2016:1481727.

13. Ye F CF, Zhang XB, Yu WM. Application and evaluation of double-needle continuous suture in urethrovesical anastomosis of laparoscopic radical prostatectomy. Medical Journal of Wuhan University 2019;40:462-6.

14. De Carlo F, Celestino F, Verri C, et al. Retropubic, laparoscopic, and robot-assisted radical prostatectomy: surgical, oncological, and functional outcomes: a systematic review. Urol Int 2014;93:373-83.

15. Zhang XP FY, Yang JJ, Wei JX. Clinical application of

Cite this article as: Li J, Zhang Q, Xie J, Yin B. Continuous poliglecaprone suture versus bidirectional barded suture for urethrovesical anastomosis during laparoscopic radical prostatectomy: a single-institute retrospective analysis of Chinese patients. Gland Surg 2020;9(5):1305-1312. doi: 10.21037/ gs-20-371 modified urethrovesical anastomosis in robot-assisted radical prostatectomy (Report of 89 cases). J Clin Urol 2017;32:445-6.

16. Takeda T, Miyajima A, Kaneko G, et al. Unidirectional barbed suture for vesicourethral anastomosis during laparoscopic radical prostatectomy. Asian J Endosc Surg 2014;7:241-5.

17. Williams SB, Alemozaffar M, Lei Y, et al. Randomized controlled trial of barbed polyglyconate versus polyglactin suture for robot-assisted laparoscopic prostatectomy anastomosis: technique and outcomes. Eur Urol 2010;58:875-81.

18. Haga N, Kurita N, Yanagida T, et al. Effects of barbed suture during robot-assisted radical prostatectomy on postoperative tissue damage and longitudinal changes in lower urinary tract outcome. Surg Endosc 2018;32:145-53. 